\title{
The importance of partnership factors and individual factors in association with absent or inconsistent condom use in heterosexuals - a cross-sectional study
}

\footnotetext{
Amy Matser ${ }^{1,2}$, Marlies Heiligenberg ${ }^{1,3}$, Ronald Geskus ${ }^{1,4}$, Titia Heijman ${ }^{1}$, Nicola Low $^{5}$, Mirjam Kretzschmar ${ }^{2,6}$, Maarten Schim van der Loeff $f^{1,3}$

Cluster of Infectious Diseases, Public Health Service of Amsterdam, the Netherlands ${ }^{1}$; Julius Centre for Health Sciences \& Primary Care, University Medical Centre Utrecht (UMCU), Utrecht, the Netherlands ${ }^{2}$; Center for Infection and Immunology Amsterdam (CINIMA), Academic Medical Center (AMC), Amsterdam, the Netherlands ${ }^{3}$; Department of Clinical Epidemiology, Biostatistics and Bioinformatics, University of Amsterdam, Amsterdam, the Netherlands ${ }^{4}$; Division of Clinical Epidemiology \& Biostatistics, Institute of Social and Preventive Medicine, University of Bern, Bern, Switzerland $^{5}$; Centre for Infectious Disease Control, National Institute for Public Health and the Environment (RIVM), Bilthoven, the Netherlands ${ }^{6}$
}

Sex Transm Infect doi:10.1136/sextrans-2013-051087

http://sti.bmj.com/content/early/2014/02/18/sextrans-2013-051087.short

\section{ACKNOWLEDGEMENTS}

The authors would like to thank the participants and M. van Rooijen for the datamanagement and $\mathrm{N}$. Wensink for data collection. We also thank two anonymous reviewers and the editors for their useful comments. This study was part of the Academic Collaborative of the Public Health Service of the City of Amsterdam (GGD) and the Academic Medical Center at the University of Amsterdam (AMC); network for infectious diseases'.

\section{FUNDING}

This work was supported by the Netherlands Organisation for Health and Research (ZonMw) [grant number 71150001] 


\begin{abstract}
Background Decisions to use condoms are made within partnerships. We examined the associations between inconsistent or no condom use and individual and partnership characteristics. We also examined the relative importance of individual versus partnership factors.

Methods Cross-sectional study of heterosexual individuals enrolled from the sexually transmitted infections (STI) outpatient clinic in Amsterdam, the Netherlands, from May to August 2010. Participants completed a questionnaire about sexual behaviour with the last four partners in the preceding year. Participant and partnership factors associated with inconsistent or no condom use in steady and casual partnerships were identified.

Results 2144 individuals were included, reporting 6401 partnerships; $54.7 \%$ were female, the median age was 25 (IQR 22-30) years and $79.9 \%$ were Dutch. Inconsistent or no condom use occurred in $13.9 \%$ of 2387 steady partnerships and in $33.5 \%$ of 4014 casual partnerships. There was statistical evidence of associations between inconsistent condom use in steady partnerships and ethnic concordance, longer duration, higher number of sex acts, practising anal sex, and sex-related drug use. In casual partnerships, associations were found with having an older partner, ethnic concordance, longer duration, higher number of sex acts, anal sex, sex-related drug use, ongoing partnerships, and concurrency. In multivariable models, partnership factors explained $50.9 \%$ of the variance in steady partnerships and $70.1 \%$ in casual partnerships compared with $10.5 \%$ and $15.4 \%$ respectively for individual factors.

Conclusion Among heterosexual STI clinic attendees in Amsterdam, partnership factors are more important factors related with inconsistent condom use than characteristics of the individual.
\end{abstract}

\title{
INTRODUCTION
}

Inconsistent condom use is common among heterosexuals $[1,2,3]$ and increases the risk of sexually transmitted infections (STI) [4]. The decision to use condoms is based on perceived risk and beliefs $[5,6]$ and also perceived willingness of the partner [1]. Individuals who use condoms inconsistently have been reported to be of older age, non-white ethnicity and lower educational level, and to report an increased number of lifetime partners, younger age at first sex, more frequent sexual contact, and drug use $[1,7,8]$. In recent years, the importance of partnership characteristics have become increasingly recognised. For example, inconsistent condom use is associated with longer partnership duration [3] and with partnerships in which women are older [9].

Previous studies have tended to focus on either individual or partnership factors associated with condom use or its absence, but their relative importance is not known. In addition, whilst it is wellestablished that condoms are more often used in new or 
casual than in longstanding partnerships, it is not known whether different factors are associated with condom use in steady partnerships and casual partnerships cassell, macaluso. The objectives of this study were to examine associations between inconsistent condom use in steady and casual heterosexual partnerships, and to examine the relative importance of individual versus partnership factors in both steady and casual partnerships.

\section{MATERIALS AND METHODS}

Study population and setting The study population was recruited from the STI clinic in Amsterdam, the Netherlands, where clients are tested and treated according to the clinic protocol $[8,11]$. Between May and August 2010, heterosexuals were invited to participate in a cross-sectional study until approximately 1000 men and 1000 women were included if they visited the clinic for a new consultation, were at least 16 years old, could understand written Dutch or English and gave written informed consent. Men who had sex with men were excluded. The study was approved by the medical ethics committee of the Academic Medical Center.

Questionnaire Participants completed an online questionnaire at the clinic, asking about their sociodemographics and sexual behaviour. The questionnaire also included questions about sexual behaviour in selfdefined steady or casual partnerships with up to four partners in the year preceding study participation and questions about partners demographics. No a priori definition of partnership status (i.e., steady or casual) was provided to allow participants to subjectively determine partnership status.

Statistical analysis The analyses were done at the partnership level: participants were represented with up to 4 partnerships. The outcome measure was inconsistent condom use within a partnership, defined as vaginal or anal sex during which condoms were not always or never used. The analysis was stratified by self-defined partner type (i.e. steady or casual). Possible associated factors were selected a priori based on prior knowledge and examined in univariable and multivariable analysis; no further selection methods, such as backward or forward selections, were used. The selected participant factors were gender, age, ethnicity, education, and number of partners in the preceding year. Education was categorised into lower (i.e. similar to elementary school, high school, or vocational training) and higher education (i.e. similar to college or university). Ethnicity was based on country of birth and country of birth of the parents; if at least one parent was born in a country other than the Netherlands the participant was regarded as belonging to a non-Dutch ethnic group. Ethnicity was then categorised into Dutch, Surinamese/Antillean, or Other.

Eight partnership factors were selected (i.e. age difference, ethnic mixing, duration, number of sex acts, anal sex, drug use, whether the partnership was ongoing, and concurrency). Age difference in years was calculated by subtracting partners age from the participants age. Drug use was defined as recreational use of XTC (3,4-methyleendioxymethamfetamine), GHB ( $\gamma$-hydroxybutyric acid), (meth)amphetamines, cocaine, poppers (amyl nitrate) or sildefanil shortly before or during sex. The questionnaire enquired about the month and year of first and last sex; it did not ask for the exact dates, as it seemed unlikely participants would remember those accurately. Also, participants were allowed to skip these questions, so as to avoid pure guessing. To calculate partnership duration we assumed that partnerships started and 
ended on the $15^{t h}$ day of the month and subtracted the first date of sex from the last date. Partnerships were chronologically ordered by date of first sex to determine whether a previous partnership was ongoing at the time the current partnership was initiated. The data available allowed concurrency to be categorised as follows: 1) definitely concurrent, when the date of first sex within a partnership was more than a month before the end of the last sex date of the previous partnership, 2) unknown, when the date of first sex and the date of last sex of the previous partnership were in the same month, 3) and definitely non-concurrent, when the date of first sex was more than a month after the end of the last sex date of the previous partnership. We assumed that behaviour within a partnership is only determined by events or partnerships in the past or present, not by future partnerships. Because only information on a maximum of four partnerships was available, concurrency was missing for most first described partnerships ( $n=2071)$, except when it was the first lifetime partnership of a participant $(n=73)$; in the latter case the partnership was definitely non-concurrent. From 2045 non-first partnerships the value of concurrency could not be estimated; this occurred if either the date of last sex of the previous partnership was missing or if the chronological order of all partnerships of a participant could not be determined.

Missing data for concurrency, drug use $(n=7,0.11 \%)$, anal sex $(n=5,0.08 \%)$, number of sex acts $(n=3,0.05 \%)$, ongoing partnership $(n=2,0.03 \%)$, and duration ( $n=1539,24 \%$ ) were imputed using multivariate imputation (MI) by chained equations.[12] The MI prediction model included all variables that were selected for the main analysis and the variables cohabiting, and number of lifetime partners. Twenty imputed data sets were created. For every data set, a logistic regression model was fitted using generalised estimating equations, to account for correlated outcomes within participants. Results from the twenty fits were pooled. Interactions between gender and other covariates were checked. Interaction terms were added if there was statistical evidence of interaction. Effects of continuous variables age, number of partners, age difference, and partnership duration were modelled via restricted cubic splines [13] with knots on the $2.5^{t h}, 25^{t h}, 50^{t h}, 75^{t h}$, and $97.5^{t h}$ percentiles. Results were compared with results from complete case analysis (Supplement). Diagnostic tests and sensitivity analysis were done to check the plausibility and robustness of the imputations (Supplement).

To estimate the relative importance of participant factors and partnership factors, we used the scaled Brier score, a measure of explained variance (i.e. between 0 and $100 \%) .[14,15]$ The Brier score is the squared difference between the predicted probability of the outcome and the observed outcome, averaged over the individuals. In the scaled Brier score, this number is scaled by its score under a noninformative model, i.e. a model that includes an intercept but no predictors. We fitted the models for steady and casual partnerships separately for individual and partnership factors. Because the scaled Brier score does not account for clustering, we randomly selected one partnership per participant and estimated the score on a dataset with one observation per participant.

Analysis was done using R 2.14.1 [16], using the packages mice [12], mitools [17], geepack [18, 19], Epi [20], and aod [21].

\section{RESULTS}


During the study period, 5120 eligible clinic visitors were invited to participate, of whom 2200 (43.0\%) gave informed consent. Compared to eligible but non-participating individuals, participants were more often of Dutch nationality (91.6\% vs. $82.9 \%)$ and more likely to have symptoms $(34.8 \%$ vs. $28.9 \%)$. Age, the number of partners in the previous six months, and STI prevalences were comparable between participants and non-participants.

Participants were excluded from further analysis if neither vaginal nor anal sex occurred with any of the partners. Specific partnerships were excluded if neither vaginal nor anal sex was reported. In total, 2144 individuals reporting 6401 partnerships were included. They reported a median of 4 (IQR 2-6) partners in the preceding year; the median number of steady partners was 1 (IQR 1-2) and of casual partners 2 (IQR 1-5). Women represented $54.7 \%$ of the study population. They were younger than men $(P<0.001)$, had more often received higher education $(P<0.001)$, and reported fewer partners $(P<0.001)$. Distributions according to ethnicity, condom use during vaginal sex and or STI diagnoses were comparable.

Participants described 2387 steady and 4014 casual partnerships. On average, casual partnership duration was shorter than steady partnership duration, and the number of sex acts was higher in steady partnerships (Table 1). Inconsistent condom use was reported in $2056(86.1 \%)$ steady partnerships; in $962(46.8 \%)$ of these partnerships condoms were never used. Inconsistent condom use was reported in 2671 (66.5\%) casual partnerships; in 1354 (50.7\%) of these partnerships condoms were never used. In univariable analysis, the odds ratio (OR) for inconsistent condom use within steady partnerships compared to casual partnerships was 3.12 (95\% CI 2.73-3.57).

Steady partnerships. In univariable analysis, low educational level was the only participant factor associated with inconsistent condom use (Table 2). All partnership factors, except whether the partnership was ongoing, were associated with inconsistent condom use (Table 2). In multivariable analysis, we found statistical evidence of an interaction between gender and ethnicity of the participant $(P=0.012)$. Dutch women were more likely to report inconsistent condom use than Dutch men. Men of non-Dutch origin were most likely to report inconsistent condom use, but the opposite effect was found in women.

At the partnership level, most associations with inconsistent condom use remained in multivariable analysis. The associations with anal sex and number of sex acts were attenuated. There was no longer statistical evidence of an association for those in definite concurrent partnerships or age difference (Table 2). The scaled Brier score of individual factors was $10.5 \%$, and the explained variance of a model with only participant characteristics was $50.9 \%$.

participant characteristics, age was the only variable for which there was strong statistical evidence of an association with inconsistent condom use in univariable analysis. There was strong statistical evidence of associations between all partnership factors, except concurrency, and inconsistent condom use (Table 3). In multivariable analysis, the association with age of the individual was attenuated $(P=0.097)$, but the trend was the same, with an increase in the aOR for inconsistent condom use for those aged 16 to 22 . Low education increased the risk of inconsistent condom use (aOR 1.33; 95\% CI 1.09-1.62). The likelihood of inconsistent condom use decreased when the number 
of partners of the individual participant increased from one to five, while from five partners onwards the aOR remained constant (overall effect $P=0.020$ ). All associations between partnership characteristics and inconsistent condom use remained. The strength of association with age difference was attenuated but inconsistent condom use was still less likely when the partner was much older $(P=0.022)$. (Table $3)$. Within a casual partnership, men were generally older than women; median age difference reported by men was 2 (IQR 0 to 5 ) years, while the age difference reported by women was -1 (IQR -4 to 1$)$ year $(P<0.001)$, but the effect of age difference on inconsistent condom use did not differ significantly for men and women. Concordant ethnicity was associated with inconsistent condom use (aOR 1.33; 95\% CI 1.14-1.56). Inconsistent condom use was also more common in longer partnerships $(P=0.003)$. A higher number of sex acts in the preceding year, anal sex, and sex-related drug use were associated with inconsistent condom use $(P<0.001)$ (Table 3$)$. Inconsistent condom use was more often practised in casual partnerships that were still ongoing at the time of inclusion (aOR 1.42; 95\% CI 1.10-1.82), while consistent condom use was more often practised in partnerships that started as concurrent partnerships (aOR $0.87 ; 95 \%$ CI $0.75-1.02$ ). Individual level factors explained $15.4 \%$ of the variance and partnership factors explained $70.1 \%$.

Sensitivity analysis In sensitivity analysis, aORs and confidence intervals compared to those obtained after MI hardly changed. In complete case analysis, 710 steady partnerships and 1607 casual partnerships were included; inconsistent condom use was reported in $83.0 \%$ and $67.1 \%$ respectively. Overall, confidence intervals became wider, but the direction of the effects and the effect sizes did not change much from those based on MI data sets. The results of complete case and sensitivity analysis are shown in the online supplement.

\section{DISCUSSION}

Among heterosexual STI clinic attendees in Amsterdam, we found that partnership factors were more important factors of inconsistent condom use than individual factors. The strongest factors associated with inconsistent condom use in steady and casual partnerships were longer partnership duration, an increased number of sex acts with a partner and practising anal sex. Inconsistent condom use was also more often reported in partnerships with concordant ethnicity and in casual partnerships in which the reported partner was older.

The strengths of this study, conducted at the largest STI clinic in the Netherlands, are the large number of partnerships described by participants and detailed information that was collected. Information was available for up to four partners per participant and included many characteristics, enabling us to study partnership characteristics and mixing by age and ethnicity. We regard the study population as a good reflection of the total eligible clinic visitor population, as there were no age differences or differences in sexual risk behavior and STI prevalence between participants and nonparticipants. Participants were more often Dutch than non-participants, which was probably due to the fact that the questionnaire was only available in Dutch and En-

glish. STI clinic visitors are, however, a high risk population so extrapolation of the results to the general heterosexual population might not be possible.

We used a self-defined partnership definition (i.e. steady or casual) to allow par- 
ticipants to subjectively determine partnership status. The advantage of using this subjective definition is that it is in accordance with the perception of the participants when deciding to use condoms or not. On the other hand, not everyone uses the same definition, which might introduce bias. For example, it is not inconceivable that women consider partnerships more often steady than men.

The most important study weakness was missing data, especially in variables measuring duration and timing of partnerships. Missing data arose because questions about first and last sex dates with each partner were not obligatory. To prevent introduction of bias we used MI [22]. Based on the results of diagnostic tests and sensitivity analyses, we are confident that MI did not introduce bias. The definition of the primary outcome, inconsistent condom use, might be considered a limitation. In approximately $50 \%$ of the partnerships with inconsistent condom use condoms were never used during sexual contact, while the remaining $50 \%$ had not always used condoms. We used this definition because one unprotected sex act can be enough to transmit an STI. This definition has been used in other studies [23, 24] and provides insight into risk behaviour during the course of the partnership. Other studies examined condom use at first and/or last sex $[3,7,25]$, which reduces errors resulting from recall bias. Recall bias might have affected our analysis, because questions were asked about self-reported behaviour in the year preceding participation and about partners details which might have been forgotten. It is unknown to what extent participants reported their partners characteristics accurately, especially in very short partnerships.

We included the four most recent partnerships in the preceding year, while $46.5 \%$ of the participants had more partners in the period than they described in detail, of whom $81.1 \%$ had just one extra partner. This sampling strategy might cause bias, because information of the other partnerships is missing and longer partnerships are more likely to be included than shorter partnerships [7].

To estimate the contribution of individual and partnership factors, we calculated scaled Brier scores using the same data set that was used in model construction. This may lead to overoptimism in model performance. However, the difference in explained variation between individual level factors and partnership factors was very large and conclusions will not be affected.

This study shows that stratification by partner type (i.e., steady or casual) was justified in views of the strong associations with patterns of condom use, partnership duration, and the number of sex acts. We found that within casual partnerships men were generally older than women and that condom use in partnerships in which the partner was older was more often inconsistent. This association was only found in casual partnerships and not in steady partnership, suggesting that differences become less important when familiarity between partners increases. This effect was already known for men [9] and we found the same effect for women.

Inconsistent condom use was more common in partnerships in which anal sex was practised. There might be various reasons for heterosexuals to have anal sex. It might be used as strategy to preserve female virginity; it prevents unwanted pregnancy; and it might be more adventurous and thus be a marker of risky behaviour. We also showed that in a population that was predominantly Dutch, concordant ethnicity within a partnership was associated with inconsistent condom use. This association has been 
shown for ethnic minorities in Amsterdam, but not yet for Dutch individuals [23, 24].

In the Netherlands, Surinamese and Antillean individuals have a higher chlamydia risk than Dutch individuals [26, 27]. Ethnic mixing patterns might help to maintain differences in chlamydia prevalence, especially because inconsistent condom use was more common in assortative partnerships [28].

For future research, we suggest that ideally studies include both members of a partnership to gain additional insights into social structures, e.g., mixing patterns by age and ethnic group, which contribute to decision-making about condom use. Increased understanding of individual and partnership factors associated with inconsistent condom use could contribute to more tailored and effective STI prevention campaigns to promote condom use.

In conclusion, we have shown that partnership factors, such as duration, the number of sex acts, and mixing by age and ethnicity, are more important factors associated with inconsistent condom use than characteristics of the individual participants and are important to consider when studying sexual risk behaviour in heterosexuals.

\section{KEY MESSAGES}

1) Partnership factors are more important factors associated with inconsistent condom use than characteristics of the individual.

2) Partnership factors, such as duration, the number of sex acts, and mixing by age and ethnicity, are important factors related with inconsistent condom in heterosexuals partnerships.

3) In future research, more emphasis on partnership characteristics is needed.

4) Studies that include both members of a partnership are recommended to gain additional insights into social structures (e.g. mixing patterns). 
Table 1 Characteristics of 4727 partnerships in which inconsistent condom use was practised and 1674 partnerships in which consistent outpatient clinic in Amsterdam in 2010

\begin{tabular}{|c|c|c|c|c|c|c|}
\hline & $\begin{array}{l}\text { Steady partnerships } \\
\text { Total } \\
(\mathrm{n}=2387)\end{array}$ & $\begin{array}{l}\text { Inconsistent } \\
\text { condom use } \\
(\mathrm{n}=2056)\end{array}$ & $\begin{array}{l}\text { Consistent } \\
\text { condom use } \\
(\mathrm{n}=331)\end{array}$ & $\begin{array}{l}\text { Casual partnerships } \\
\text { Total } \\
(\mathrm{n}=4014)\end{array}$ & $\begin{array}{l}\text { Inconsistent } \\
\text { condom use } \\
(\mathrm{n}=2671)\end{array}$ & $\begin{array}{l}\text { Consistent } \\
\text { condom use } \\
(\mathrm{n}=1343)\end{array}$ \\
\hline \multicolumn{7}{|l|}{ Participant characteristics } \\
\hline Female gender & $1293(54.2 \%)$ & $1114(54.2 \%)$ & $179(54.1 \%)$ & $2016(50.2 \%)$ & $1380(51.7 \%)$ & $636(47.4 \%)$ \\
\hline \multicolumn{7}{|l|}{ Ethnicity } \\
\hline Dutch & $1875(78.6 \%)$ & $1618(78.7 \%)$ & $257(77.6 \%)$ & $3297(82.1 \%)$ & $2224(83.3 \%)$ & $1073(79.9 \%)$ \\
\hline Surinamese/Antillean & $241(10.1 \%)$ & $211(10.3 \%)$ & $30(9.1 \%)$ & $296(7.4 \%)$ & $181(6.8 \%)$ & $115(8.6 \%)$ \\
\hline Other & $271(11.4 \%)$ & $227(11.0 \%)$ & $44(13.3 \%)$ & $420(10.5 \%)$ & $265(9.9 \%)$ & $155(11.5 \%)$ \\
\hline Median age in years (IQR) & $25(22-30)$ & $25(22-30)$ & $25(23-30)$ & $25(22-29)$ & $25(22-29)$ & $25(23-31)$ \\
\hline Low educational level & $796(33.3 \%)$ & $712(34.6 \%)$ & $84(25.4 \%)$ & $1221(30.4 \%)$ & $826(30.9 \%)$ & $395(29.4 \%)$ \\
\hline Median no. of partners in the preceding year (IQR) & $4(2-6)$ & $4(2-6)$ & $4(3-7)$ & $5(4-8)$ & $5(4-8) 5(4-8)$ & \\
\hline \multicolumn{7}{|l|}{ Characteristics of the partnership } \\
\hline Median age difference in years (IQR) ${ }^{a}$ & $0(-3-3)$ & $0(-3-3)$ & $0(-2-3)$ & $0(-2-3)$ & $0(-2-3)$ & $0(-3-4)$ \\
\hline Discordant ethnic mixing & $788(33.0 \%)$ & $655(31.9 \%)$ & $133(40.2 \%)$ & $1478(36.8 \%)$ & $890(33.3 \%)$ & $588(43.8 \%)$ \\
\hline \multirow{2}{*}{\multicolumn{7}{|c|}{ Number of sex acts within a partnership in the preceding year }} \\
\hline & & & & & & \\
\hline $1-4$ & $364(15.3 \%)$ & $280(13.6 \%)$ & $84(25.4 \%)$ & $2953(73.6 \%)$ & $1824(68.3 \%)$ & $1129(84.1 \%)$ \\
\hline $5-19$ & $671(28.1 \%)$ & $543(26.4 \%)$ & $128(38.7 \%)$ & $790(19.7 \%)$ & $620(23.2 \%)$ & $170(12.7 \%)$ \\
\hline $20-39$ & $475(19.9 \%)$ & $409(19.9 \%)$ & $66(19.9 \%)$ & $166(4.1 \%)$ & $137(5.1 \%)$ & $29(2.2 \%)$ \\
\hline$\geq 40$ & $876(36.7 \%)$ & $823(40.1 \%)$ & $53(16.0 \%)$ & $103(2.6 \%)$ & $88(3.3 \%)$ & $15(1.1 \%)$ \\
\hline Anal sex ${ }^{b}$ & $455(19.1 \%)$ & $425(20.7 \%)$ & $30(9.1 \%)$ & $249(6.2 \%)$ & $204(7.7 \%)$ & $45(3.4 \%)$ \\
\hline Sex-related drug use $e^{b, c}$ & $1463(61.4 \%)$ & $1299(63.3 \%)$ & $164(49.6 \%)$ & $2490(62.1 \%)$ & $1760(66.0 \%)$ & $730(54.4 \%)$ \\
\hline Partnership ongoing at the time of inclusion ${ }^{b}$ & $1184(49.6 \%)$ & $1035(50.4 \%)$ & $149(45.0 \%)$ & $523(13.0 \%)$ & $403(15.1 \%)$ & $120(8.9 \%)$ \\
\hline \multicolumn{7}{|l|}{ Concurrency of partnership ${ }^{d}$} \\
\hline Definitely non-concurrent & $804(33.7 \%)$ & $663(32.2 \%)$ & $141(42.6 \%)$ & $1326(33.0 \%)$ & $905(33.9 \%)$ & $421(31.3 \%)$ \\
\hline Unknown & $198(8,3 \%)$ & $174(8.5 \%)$ & $24(7.3 \%)$ & $602(15.0 \%)$ & $383(14.3 \%)$ & $219(16.3 \%)$ \\
\hline Definitely concurrent & $1385(58.0 \%)$ & $1219(59.3 \%)$ & $166(50.2 \%)$ & $2086(52.0 \%)$ & $1383(51.8 \%)$ & $703(52.3 \%)$ \\
\hline
\end{tabular}

IQR $=$ interquartile range. ${ }^{a}$ Age of the partner subtracted from age of the participant; ${ }^{b}$ The number of missings per variable were 1539 for duration, 3 for number of sex acts, 5 for anal sex, 7 for recreational drug use, 2 for ongoing partnership ; ${ }^{c}$ Recreational use of XTC, GHB, amphetamines, cocaine, poppers, or sildefanil prior to or during sex; ${ }^{d}$ For people reporting $>4$ eligible partners, concurrency could not be established for the first of four reported partners for these, concurrency data was estimated after multiple imputation. For details, see appendix 
Table 2: Factors associated with inconsistent condom use in 2387 steady partnerships, among 1705 heterosexuals attending the sexually transmitted infections outpatient clinic, Amsterdam, 2010

\begin{tabular}{|c|c|c|c|c|}
\hline & OR (95\% CI) & p-value & aOR $(95 \% \mathrm{CI})$ & p-value \\
\hline \multicolumn{5}{|l|}{ Individual participant characteristics } \\
\hline Gender & & 0.940 & & \\
\hline Male & 1 & & & \\
\hline Female & $1.01(0.78-1.31)$ & & & \\
\hline Ethnicity & & 0.290 & & \\
\hline Dutch & 1 & & & \\
\hline Surinamese/Antillean & $1.14(0.72-1.81)$ & & & \\
\hline Other & $0.76(0.51-1.12)$ & & & \\
\hline Interaction between ethnicity and gender & & & & 0.012 \\
\hline Dutch males & & & 1 & \\
\hline Surinamese/Antillean males & & & $2.27(1.07-4.82)$ & \\
\hline Other males & & & $2.19(1.12-4.29)$ & \\
\hline Dutch females & & & $1.35(0.94-1.94)$ & \\
\hline Surinamese/Antillean females & & & $1.15(0.56-2.36)$ & \\
\hline Other females & & & $0.72(0.40-1.29)$ & \\
\hline Age in years ${ }^{a}$ & $* a$ & 0.530 & & 0.370 \\
\hline 20 & $1.30(0.91-1.84)$ & & $1.41(0.94-2.12)$ & \\
\hline 25 & $1.17(0.94-1.45)$ & & $1.14(0.90-1.44)$ & \\
\hline 30 & 1 & & 1 & \\
\hline 35 & $0.93(0.77-1.13)$ & & $0.95(0.77-1.19)$ & \\
\hline 40 & $0.94(0.72-1.23)$ & & $1.01(0.75-1.37)$ & \\
\hline Education & & 0.002 & & $<0.001$ \\
\hline High & 1 & & 1 & \\
\hline Low & $1.57(1.17-2.09)$ & & $1.96(1.38-2.78)$ & \\
\hline No. of partners in the year preceding study participation $a$ & & 0.540 & & 0.640 \\
\hline 1 & 1 & & 1 & \\
\hline 2 & $0.95(0.56-1.60)$ & & $1.28(0.73-2.25)$ & \\
\hline 4 & $0.79(0.48-1.31)$ & & $1.01(0.58-1.76)$ & \\
\hline 8 & $0.72(0.43-1.20)$ & & $0.95(0.53-1.68)$ & \\
\hline \multicolumn{5}{|l|}{ Characteristics of the partnership } \\
\hline Age difference in years ${ }^{a, b}$ & $* a$ & 0.007 & $* a$ & 0.061 \\
\hline-10 & $1.47(0.89-4.45)$ & & $1.47(0.84-2.57)$ & \\
\hline-5 & $1.01(0.70-1.46)$ & & $0.99(0.68-1.45)$ & \\
\hline 0 & 1 & & 1 & \\
\hline 5 & $1.00(0.72-1.38)$ & & $1.15(0.79-1.69)$ & \\
\hline 10 & $0.80(0.54-1.19)$ & & $0.96(0.60-1.54)$ & \\
\hline Ethnic mixing & & 0.005 & & 0.012 \\
\hline Discordant & 1 & & 1 & \\
\hline Concordant & $1.45(1.12-1.89)$ & & $1.46(1.09-1.95)$ & \\
\hline
\end{tabular}




\begin{tabular}{lllll} 
Duration of a partnership in days ${ }^{a, c}$ & $* a$ & $<0.001$ & $* a$ & $<0.001$ \\
1 & 1 & & 1 & \\
{$[1,30]$} & $1.94(1.30-2.92)$ & & $2.20(1.45-3.36)$ & \\
{$[1,60]$} & $2.36(1.60-3.46)$ & & $2.35(1.56-3.53)$ & \\
{$[210,270]$} & $4.20(2.79-6.30)$ & & $3.20(2.04-5.02)$ & \\
Number of sex acts within a partnership in the preceding year & & $<0.001$ & & \\
$1-4$ & 1 & & 1 & \\
$5-19$ & $1.31(0.95-1.80)$ & & $1.02(0.72-1.44)$ & \\
$20-39$ & $1.93(1.35-2.77)$ & & $2.77(0.93-2.01)$ & \\
$\geq 40$ & $4.88(3.36-7.09)$ & & $2.76-4.15)$ & \\
Anal sex & $2.57(1.70-3.90)$ & $<0.001$ & $1.67(1.09-2.57)$ & 0.019 \\
Sex-related drug use d & $1.83(1.42-2.35)$ & $<0.001$ & $1.71(1.30-2.26)$ & $<0.001$ \\
Partnership is ongoing at the time of inclusion & $1.21(0.94-1.56)$ & 0.140 & $1.04(0.79-1.37)$ & 0.800 \\
Concurrency of partnership & & 0.015 & 1 & 0.550 \\
Definitely non-concurrent & 1 & & $1.30(0.81-2.08)$ & \\
Unknown & $1.73(0.98-3.07)$ & & $1.03(0.75-1.40)$ & \\
Definitely concurrent & $1.43(1.10-1.85)$ & & & \\
\hline
\end{tabular}

$\mathrm{OR}=$ odds ratio; $\mathrm{CI}=$ confidence interval; aOR = adjusted odds ratio. NOTE. Missing data in duration, number of sex acts, anal sex, recreational drug use, ongoing partnership, and concurrency of partnership were imputed. ${ }^{a}$ Continuous variables age in years, number of partners in the preceding 6 months, age difference in years, and duration in days are modelled as restricted cubic splines with nodes on the $2.5^{t} h, 25^{t} h, 50^{t} h, 75^{t} h$, and $97.5^{t} h$ percentiles; example odds ratios are given; ${ }^{b}$ Age of the partner subtracted from age of the participant; ${ }^{c}$ Only the month and year of first and last sexual contact were known, causing uncertainty in duration of the partnership; ${ }^{d}$ Recreational use of XTC, GHB, amphetamines, cocaine, poppers, or sildefanil prior to or during sex; ${ }^{e}$ Only the month and year of first and last sexual contact of partnerships were known. For partnerships with an unknown concurrency status, concurrency could not be determined accurately. 
Table 3: Factors associated with inconsistent condom use in 4014 casual partnerships, among 1707 heterosexuals attending the sexually transmitted infections outpatient clinic, Amsterdam, 2010

\begin{tabular}{|c|c|c|c|c|}
\hline & OR $(95 \% \mathrm{CI})$ & p-value & aOR $(95 \% \mathrm{CI})$ & p-value \\
\hline \multicolumn{5}{|l|}{ Individual participant characteristics } \\
\hline Gender & & 0.061 & & 0.140 \\
\hline Male & 1 & & 1 & \\
\hline Female & $1.17(0.99-1.37)$ & & $1.15(0.95-1.39)$ & \\
\hline Ethnicity & & 0.098 & & 0.320 \\
\hline Dutch & 1 & & 1 & \\
\hline Surinamese/Antillean & $0.77(0.58-1.03)$ & & $0.79(0.58-1.08)$ & \\
\hline Other & $0.83(0.64-1.07)$ & & $1.02(0.78-1.34)$ & \\
\hline Age in years ${ }^{a}$ & & 0.003 & & 0.097 \\
\hline 20 & $1.08(0.87-1.35)$ & & $1.17(0.92-1.48)$ & \\
\hline 25 & $1.08(0.92-1.26)$ & & $1.10(0.94-1.28)$ & \\
\hline 30 & 1 & & 1 & \\
\hline 35 & $0.90(0.81-1.03)$ & & $0.93(0.81-1.07)$ & \\
\hline 40 & $0.79(0.67-0.94)$ & & $0.85(0.69-1.04)$ & \\
\hline Education & & 0260 & & 0.005 \\
\hline High & 1 & & 1 & \\
\hline Low & $1.11(0.93-1.32)$ & & $1.33(1.09-1.62)$ & \\
\hline No. of partners in the year preceding study participation $a$ & & 0.160 & & 0.020 \\
\hline 1 & 1 & & 1 & \\
\hline 2 & $0.70(0.45-1.09)$ & & $0.77(0.59-0.99)$ & \\
\hline 4 & $0.52(0.27-1.03)$ & & $0.65(0.49-0.85)$ & \\
\hline 8 & $0.53(0.29-0.98)$ & & $0.66(0.48-0.89)$ & \\
\hline \multicolumn{5}{|l|}{ Characteristics of the partnership } \\
\hline Age difference in years ${ }^{a, b}$ & $* a$ & $<0.001$ & $* a$ & 0.18 \\
\hline-10 & $0.80(0.64-1.01)$ & & $0.72(0.57-0.91)$ & \\
\hline-5 & $0.80(0.66-0.96)$ & & $0.75(0.62-0.91)$ & \\
\hline 0 & 1 & & 1 & \\
\hline 5 & $0.94(0.79-1.13)$ & & $1.00(0.81-1.24)$ & \\
\hline 10 & $0.77(0.61-0.97)$ & & $0.89(0.68-1.18)$ & \\
\hline Ethnic mixing & & $<0.001$ & & $<0.001$ \\
\hline Discordant & 1 & & 1 & \\
\hline Concordant & $1.40(1.21-1.61)$ & & $1.33(1.14-1.56)$ & \\
\hline Duration of a partnership in days ${ }^{a, c}$ & $* a$ & ??? & $* a$ & ??? \\
\hline 1 & 1 & & 1 & \\
\hline$[1,30]$ & $1.67(1.40-2.00)$ & & $0.90(0.50-1.61)$ & \\
\hline$[1,60]$ & $1.70(1.46-1.98)$ & & $1.06(0.69-1.61)$ & \\
\hline$[210,270]$ & $1.76(1.48-2.09)$ & & $1.38(1.10-1.72)$ & \\
\hline Number of sex acts within a partnership in the preceding year & & $<0.001$ & & $<0.001$ \\
\hline $1-4$ & 1 & & 1 & \\
\hline
\end{tabular}




\begin{tabular}{|c|c|c|c|c|}
\hline $5-19$ & $2.14(1.78-2.57)$ & & $1.78(1.46-2.17)$ & \\
\hline $20-39$ & $3.19(2.18-4.66)$ & & $2.65(1.78-3.95)$ & \\
\hline$\geq 40$ & $3.95(2.25-6.95)$ & & $2.78(1.55-4.99)$ & \\
\hline Anāl sex & $2.53(1.79-3.58)$ & $<0.001$ & $2.12(1.48-3.05)$ & $<0.001$ \\
\hline Sex-related drug use d & $1.60(1.40-1.86)$ & $<0.001$ & $1.56(1.33-1.81)$ & $<0.001$ \\
\hline Partnership is ongoing at the time of inclusion & $1.99(1.59-2.49)$ & $<0.001$ & $1.42(1.10-1.82)$ & 0.007 \\
\hline Concurrency of partnership ${ }^{e}$ & & 0.069 & & 0.027 \\
\hline Definitely non-concurrent & 1 & & 1 & \\
\hline Unknown & $0.81(0.67-0.98)$ & & $0.77(0.64-0.94)$ & \\
\hline Definitely concurrent & $0.89(0.78-1.02)$ & & $0.87(0.75-1.02)$ & \\
\hline
\end{tabular}

$\mathrm{OR}=$ odds ratio; $\mathrm{CI}=$ confidence interval; aOR = adjusted odds ratio. NOTE. Missing data in duration, number of sex acts, anal sex, recreational drug use, ongoing partnership, and concurrency of partnership were imputed. ${ }^{a}$ Continuous variables age in years, number of partners in the preceding 6 months, age difference in years, and duration in days are modelled as restricted cubic splines with nodes on the $2.5^{t} h, 25^{t} h, 50^{t} h, 75^{t} h$, and $97.5^{t} h$ percentiles; example odds ratios are given; ${ }^{b}$ Age of the partner subtracted from age of the participant; ${ }^{c}$ Only the month and year of first and last sexual contact were known, causing uncertainty in duration of the partnership; ${ }^{d}$ Recreational use of XTC, GHB, amphetamines, cocaine, poppers, or sildefanil prior to or during sex; ${ }^{e}$ Only the month and year of first and last sexual contact of partnerships were known. For partnerships with an unknown concurrency status, concurrency could not be determined accurately. 


\section{References}

[1] Shih SL, Kebodeaux CA, Secura GM, et al. Baseline correlates of inconsistent and incorrect condom use among sexually active women in the contraceptive CHOICE Project. Sex Transm Dis 2011;38:1012-9.

[2] Eaton DK, Kann L, Kinchen S, et al. Youth risk behavior surveillance - United States, 2009. MMWR Surveill Summ 2010;59:1-142.

[3] Cassell JA, Mercer CH, Imrie J, et al. Who uses condoms with whom? Evidence from national probability sample surveys. Sex Transm Infect 2006;82:467-73.

[4] Warner L, Stone KM, Macaluso M, et al. Condom use and risk of gonorrhea and Chlamydia: a systematic review of design and measurement factors assessed in epidemiologic studies. Sex Transm Dis 2006;33:36-51.

[5] Green J, Fulop N, Kocsis A. Determinants of unsafe sex in women. Int J STD AIDS 2000;11:777-83.

[6] Flood M. Lust, trust and latex: Why young heterosexual men do not use condoms. Culture, Health \& Sexuality 2003;5:353-69.

[7] Copas AJ, Mercer CH, Farewell VT, et al. Recent heterosexual partnerships and patterns of condom use: a weighted analysis. Epidemiology 2009;20:44-51.

[8] Heiligenberg M, Wermeling PR, van Rooijen MS, et al. Recreational Drug Use During Sex and Sexually Transmitted Infections Among Clients of a City Sexually Transmitted Infections Clinic in Amsterdam, The Netherlands. Sex Transm Dis 2012;39:518-27.

[9] Manlove J, Ikramullah E, Terry-Humen E. Condom use and consistency among male adolescents in the United States. J Adolesc Health 2008;43:325-33.

[10] Macaluso M, Demand MJ, Artz LM, et al. Partner type and condom use. AIDS 2000;14:537-46.

[11] Heijman RL, Stolte IG, Thiesbrummel HF, et al. Opting out increases HIV testing in a large sexually transmitted infections outpatient clinic. Sex Transm Infect 2009;85:249-55.

[12] Van Buuren S, Groothuis-Oudshoorn K. MICE: Multivariate imputation by chained equations in R. J Stat Soft 2011;45:1-67.

[13] Durrleman S, Simon R. Flexible regression models with cubic splines. Stat Med 1989;8:551-61.

[14] Brier GW. Verification of forecasts expressed in terms of probability. Monthly Weather Review 1950;78:1-3.

[15] Steyerberg EW, Vickers AJ, Cook NR, et al. Assessing the performance of prediction models: a framework for traditional and novel measures. Epidemiology 2010;21:128-38.

[16] R Development Core Team. R: A Language and Environment for Statistical Computing. Vienna Austria: R Foundation for Statistical Computing 2011.

[17] Lumley T. mitools: Tools for multiple imputation of missing data. Vienna, Austria: R Foundation for Statistical Computing 2010.

[18] Højsgaard S, Halekoh U, Yan J. The R package geepack for Generalized Estimating Equations. J Stat Soft 2005;15:1-11. Denk aan het gekke teken in de naam Hojsgaard !!!! 
[19] Yan J. geepack: Yet another package for Generalized Estimating Equations. R News 2012;2:12-4.

[20] Carstensen B, Plummer M, Laara E, et al. Epi: A package for statistical analysis in epidemiology. Vienna Austria: R Foundation for Statistical Computing 2010.

[21] Lesnoff M, Lancelot R. aod: Analysis of Overdispersed Data. Vienna, Austria: R Foundation for Statistical Computing 2010.

[22] Donders AR, van der Heijden GJ, Stijnen T, et al. Review: a gentle introduction to imputation of missing values. J Clin Epidemiol 2006;59:1087-91.

[23] Wiggers LC, de Wit JB, Gras MJ, et al. Risk behavior and social-cognitive determinants of condom use among ethnic minority communities in Amsterdam. AIDS Educ Prev 2003;15:430-47.

[24] Gras MJ, van Benthem BH, Coutinho RA, et al. Determinants of high-risk sexual behavior among immigrant groups in Amsterdam: implications for interventions. $\mathrm{J}$ Acquir Immune Defic Syndr 2001;28:166-72.

[25] Mercer CH, Copas AJ, Sonnenberg P, et al. Who has sex with whom? Characteristics of heterosexual partnerships reported in a national probability survey and implications for STI risk. Int J Epidemiol 2009;38:206-14.

[26] Gras MJ, Weide JF, Langendam MW, et al. HIV prevalence, sexual risk behaviour and sexual mixing patterns among migrants in Amsterdam, The Netherlands. AIDS 1999;13:1953-62.

[27] Van Veen MG, Koedijk FD, van der Sande MA. STD coinfections in The Netherlands: Specific sexual networks at highest risk. Sex Transm Dis 2010;37:416-22.

[28] Matser A, Luu N, Geskus R, et al. Higher Chlamydia trachomatis prevalence in ethnic minorities does not always reflect higher sexual risk behaviour. Plos One $2013 ;$ accepted]. 